Binghamton University

The Open Repository @ Binghamton (The ORB)

Mechanical Engineering Faculty Scholarship

Mechanical Engineering

2020

\title{
Modeling and Characterization of A Pull-in Free MEMS \\ Microphone
}

Mehmet Ozdogan

Binghamton University--SUNY, mozdoga1@binghamton.edu

Shahrzad Towfighian

Binghamton University--SUNY, stowfigh@binghamton.edu

Ronald Miles

Binghamton University--SUNY, rmiles@binghamton.edu

Follow this and additional works at: https://orb.binghamton.edu/mechanical_fac

Part of the Mechanical Engineering Commons

\section{Recommended Citation}

Ozdogan, Mehmet; Towfighian, Shahrzad; and Miles, Ronald, "Modeling and Characterization of A Pull-in Free MEMS Microphone" (2020). Mechanical Engineering Faculty Scholarship. 28.

https://orb.binghamton.edu/mechanical_fac/28

This Article is brought to you for free and open access by the Mechanical Engineering at The Open Repository @ Binghamton (The ORB). It has been accepted for inclusion in Mechanical Engineering Faculty Scholarship by an authorized administrator of The Open Repository @ Binghamton (The ORB). For more information, please contact ORB@binghamton.edu. 


\title{
Modeling and Characterization of A Pull-in Free MEMS Microphone
}

\author{
Mehmet Ozdogan, Shahrzad Towfighian and Ronald N. Miles
}

\begin{abstract}
In this study, we examine the feasibility of designing a MEMS microphone employing a levitation based electrode configuration. This electrode scheme enables capacitive MEMS sensors that could work for large bias voltages without pullin failure. Our experiments and simulations indicate that it is possible to create robust sensors properly working at high DC voltages, which is not feasible for most of the conventional parallel plate electrode-based micro-scale devices. In addition, the use of larger bias voltages will improve signal-to-noise ratios in MEMS sensors because it increases the signal relative to the noise in read-out circuits. This study presents the design, fabrication, and testing of a capacitive microphone, which is made of approximately $2 \mu \mathrm{m}$ thick highly-doped polysilicon as a diaphragm. It has approximately $1 \mathrm{~mm}^{2}$ surface area and incorporates interdigitated sensing electrodes on three of its sides. Right underneath these moving electrodes, there are fixed fingers having held at the same voltage potential as the moving electrodes and separated from them with a $2 \mu \mathrm{m}$ thick air gap. The electronic output is obtained using a charge amplifier. Measured results obtained on three different microphone chips using bias voltages up to 200 volts indicate that pull-in failure is completely avoided. The sensitivity of this initial design was measured to be $16.1 \mathrm{mV} / \mathrm{Pa}$ at $200 \mathrm{~V}$ bias voltage, and the bandwidth was from $100 \mathrm{~Hz}$ to $4.9 \mathrm{kHz}$.
\end{abstract}

\section{INTRODUCTION}

Advances in consumer products and environmental noise cancellation applications have increased the demand for high sensitivity miniature microphones. Advancing micro and nanofabrication methods encouraged the key market players to replace conventional electret microphones with miniature micro-electro-mechanical-systems (MEMS) microphones. These devices are acoustic sensors working in the audible range $(20 \mathrm{~Hz}-20 \mathrm{kHz})$ and are integrated into a broad range of electronic products due to their small size, low cost, high sensitivity, and low power consumption. Today, many types of MEMS microphones can be found in smartphones, laptops, hearing aids, smart speakers, and wearables. A fully packaged MEMS microphone consists of an electronic read-out circuitry chip, a MEMS chip, a metal shielding cover, and a printed circuit board with electronic components. The vast majority of MEMS microphones use capacitive transduction, which is compatible with current fabrication technology and can achieve adequate performance in terms of low-noise and high sensitivity. The performance of capacitive microphones can be adversely affected by parasitic capacitance and nonlinearity in the diaphragm response and the capacitive transduction [1]. In this study, we focus on the design of an omnidirectional

M. Ozdogan, S. Towfighian, and R. N. Miles are with the Department of Mechanical Engineering, State University of New York at Binghamton, Binghamton, NY, 13902 USA, Corresponding e-mail: (miles@binghamton.edu) capacitive microphone that employs a novel capacitive readout scheme that differs substantially from that used in existing designs. As described below, this approach avoids the typical capacitive design constraints required to avoid the well-known pull-in instability.

Royer et al. [2] presented the first MEMS microphone in 1983. It then took almost two decades to release the first commercial MEMS microphone, which was launched by Knowles (SiSonic) and has been integrated into billions of devices. The success of this product and the potential for market growth has raised the attention given to miniature microphones by device designers. There are a number of literature reviews on MEMS microphones. Shah et al. [3] very recently published a detailed survey of this subject. A key design goal of most of the published microphones is to achieve high sensitivity, which is typically measured as the output voltage per pascal of detected sound pressure. In capacitive microphones, this electronic sensitivity is generally proportional to the applied bias voltage [4]. In 2007, Dehe et al. [5] presented a high sensitivity capacitive MEMS microphone with a measured sensitivity of $7.9 \mathrm{mV} / \mathrm{Pa}$ at $2 \mathrm{~V}$ bias voltage. The bandwidth of the device was around $100 \mathrm{~Hz}$ to $14 \mathrm{kHz}$. This study also discussed the effect of back-volume size, and it was mentioned that having small back-volume decreased the sensitivity significantly up to $11 \mathrm{~dB}$. In 2011, Chan et al. [6] presented another MEMS microphone with a sensitivity of $12.6 \mathrm{mV} / \mathrm{Pa}$. One of the very latest studies was done by Ganji et al [7]. The presented opencircuit sensitivity was around $2.46 \mathrm{mV} / \mathrm{Pa}$ at $1 \mathrm{kHz}$ with a bias voltage of $5 \mathrm{~V}$. The pull-in voltage of the device was around $10.3 \mathrm{~V}$, and the authors concluded that a low cost smallest microphone diaphragm with good performance was presented.

The acoustical sensitivity of a MEMS capacitive microphone is the measure of output voltage relative to input pressure (Volt/ $\mathrm{Pa}, \mathrm{dBV} / \mathrm{Pa}$, etc.). The response of an ideal MEMS microphone is expected to be frequency and bias voltage-independent. Also, its resonance frequency is expected to be above the audible frequency range. However, having all these features on one design has been the subject of extensive research for many decades. For example, the electrostatic force has inherent nonlinear behavior that shifts the effective stiffness of a capacitive sensor device as a function of applied bias voltage. This phenomenon is called electrostatic stiffening or the softening effect, which significantly impacts the response of the device.

The overall sensitivity of a nondirectional capacitive microphone can be improved with an increase in the applied bias voltage, area of the diaphragm, and back volume with a decrease in stiffness (resonance frequency), and the initial 
fabrication gap [8]. However, it is not trivial to adjust these parameters at will because of the technological limitations and coupled relationship between the parameters. For example, the size of the back volume is typically limited due to a desire to minimize the overall package size. Another design parameter is the effective area of the diaphragm, which can significantly affect the sensitivity as larger areas improve response. However, researchers are unable to increase the diaphragm area significantly as devices get smaller and smaller.

Capacitive sensors use electrical biasing to convert mechanical vibrations into electrical signals. Thus, a bias voltage is necessary in order to obtain an electronic readout of the diaphragm motion. The bias voltage has a direct impact to yield better sensitivity in microphones because it enhances the signal quality and increases the signal-to-noise ratio. However, it is coupled with the stiffness of the system and playing with it does not only change the sensitivity but also tunes the effective stiffness of the system. For conventional parallel plate electrode configurations, bias voltage decreases the effective stiffness (spring softening effect). This improves the sensitivity up to some point. However, when the restoring spring force weakens sufficiently, the electrostatic force can snap the diaphragm into the back-plate, a phenomenon called the pull-in effect or pull-in instability. This threshold voltage is the highest possible bias that allows a microphone to function properly.

The pull-in effect has been considered as a critical design parameter for the vast majority of capacitive MEMS devices such as accelerometers, gyroscopes, and microphones. The pull-in is a quasi-static instability that is severely limiting the performance of electronic devices having electrostatically driven MEMS devices. These devices usually consist of fixed and moving electrodes separated by an air gap. The instability occurs when the electrostatic force exceeds the elastic restoring forces after a certain amount of voltage is applied. The pull-in point for a parallel-plate capacitor could be estimated using the simple equation $V_{p}=\sqrt{\frac{8 k h^{3}}{27 \epsilon A}}$, where $k$ is the stiffness, $h$ is the air gap between the electrodes, $\epsilon$ is the permittivity, $A$ is the surface area of the electrode. According to this formula, stiffness, initial gap, and the area of the moving electrode define the pull-in point. Thus, the MEMS capacitive microphones are designed around the pull-in point, which limits increasing the sensitivity. Over two decades, researchers presented several design approaches to avoid pull-in instability. The most commonly used one is no back-plate approaches such as interdigitated comb-drive designs [9].

The major contribution of the proposed MEMS microphone is its unique sensing mechanism. The presented device uses an unconventional electrode arrangement, which has been presented in the literature as either repulsive force or levitation based electrode configuration [10], [11]. The significant benefit of this electrode scheme is the ability to avoid pull-in instability and micro-welding, which are two failure modes of electrostatic MEMS devices that cause irreversible damages. The new electrode design employs bias voltage to provide an upward electrostatic force pushing the moving electrode away to create a larger gap between the moving and fixed electrodes,

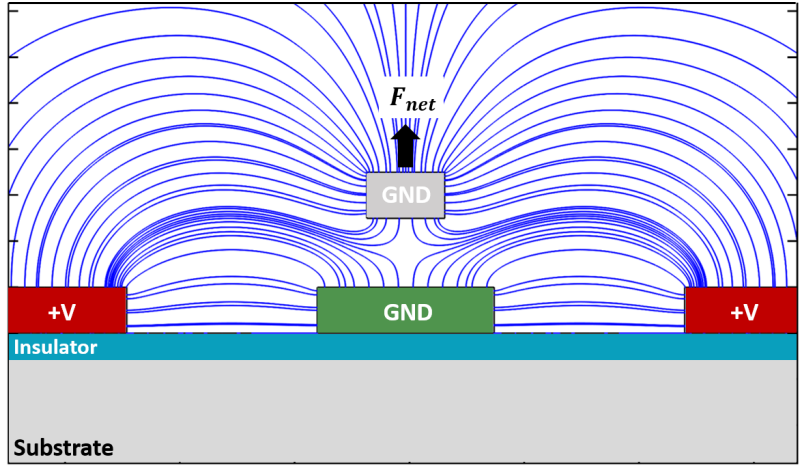

Fig. 1: The figure shows the electric field of the proposed electrode design for a unit cell. $F_{\text {net }}$ represents the total net force that is pushing the moving electrode away from the substrate.

which eliminates the possibility of the pull-in instability as well as increasing the travel range of the microphone. The electrode configuration was studied previously for actuator and sensor applications [10], [12]. However, these studies mostly exploited the mechanical response of these devices when used for actuation. Besides, we presented a numerical study [13] and preliminary experimental work [14] on a MEMS microphone design using the same electrostatic levitation design. The numerical study was a feasibility investigation on the design and fabrication parameters of the microphone. In the latter work, we presented the fabrication and initial tests of the prototype microphone. In the present study, we thoroughly evaluate the performance of the microphone, provide a simple mathematical model, and then discuss a possible future design.

\section{Device Fabrication}

The fabrication of the device started with four-inch n-type $(1-20 \Omega \mathrm{cm})$ silicon wafers. The steps of the fabrication is provided in Figure 2. The first step of the fabrication was growing a $1 \mu \mathrm{m}$ thick oxide film using a low-pressure chemical vapor deposition (LPCVD) furnace at $1100{ }^{\circ} \mathrm{C}$. On top of this insulation layer, we deposited $0.2 \mu \mathrm{m}$ thick low-stress LPCVD silicon nitride at $800{ }^{\circ} \mathrm{C}$. The insulation layers separate the microphone from the silicon foundation. On top of these layers, $2 \mu \mathrm{m}$ thick in-situ phosphorus-doped amorphous silicon was deposited in an LPCVD furnace at 570 ${ }^{\circ} \mathrm{C}$. Following the deposition, the wafers were annealed for about 300 minutes in an Argon annealing furnace at $1000{ }^{\circ} \mathrm{C}$. The annealing step forms the amorphous silicon to polysilicon and reduces both residual stresses and the resistivity of the film. After the annealing procedure, the polysilicon layer was exposed and etched using a deep reactive ion etching (DRIE) Bosch fluorine-based processing tool to create the fixed electrodes. Because the Bosch process forms a polymer layer on the sides of the etched surface, the wafers were cleaned using EKC265 post-etch residue remover for an hour at $70{ }^{\circ} \mathrm{C}$. Then a $4 \mu \mathrm{m}$ thick high-temperature-oxide (HTO) sacrificial layer was deposited in three steps at $800{ }^{\circ} \mathrm{C}$. This layer defines the air gap between fixed and moving electrodes. 
Following this process, we annealed the wafers at $425^{\circ} \mathrm{C}$ in a nitrogen annealing tube for 120 minutes. After the annealing run was completed, we planarized about the half of the oxide layer to obtain a flat surface and $2 \mu \mathrm{m}$ thick air-gap between the moving and fixed electrodes. On top of the oxide layer vias are opened using an Inductively Coupled Plasma Reactive Ion Etching (ICP-RIE) tool. This etching process uses a $\mathrm{CHF}_{3}$ and $\mathrm{O}_{2}$ gas mixture to etch the oxide anisotropically. This chemistry also forms a polymer layer, which needs to be removed using oxygen plasma or other techniques. The wafers, free of any polymer residue, were deposited with the second layer of $2 \mu \mathrm{m}$ thick phosphorus-doped polysilicon. A lithography, etching, and post-etch cleaning steps were performed to form the polysilicon diaphragm attached moving electrodes. On top of this layer, we deposited $2.5 \mu \mathrm{m}$ thick phosphosilicate glass (PSG) using a plasma-enhanced chemical vapor deposition (PECVD) tool at $350{ }^{\circ} \mathrm{C}$. This film mechanically supports the very thin diaphragm during the backside processing of the wafer. The backside polysilicon was removed using $\mathrm{KOH}$. Then, we spin-coated the backside of the wafers with primer and a positive tone resist, which was soft baked and developed to etch the thick oxide mask layer. Because the backside oxide layer was thick we used ICP tool for the etching. Following the oxide etching process, the bulk silicon was etched using DRIE silicon etcher. After the silicon etching was completed, the front sides of the wafers were spin-coated with photoresist to protect the diaphragms during the dicing process. The size of the diced chips was $2.4 \mathrm{~mm} \mathrm{X} 2.4 \mathrm{~mm} \mathrm{X} 0.5 \mathrm{~mm}$. Then, backside insulation layers were also etched using an ICP RIE process. After the backside layer etching was fully completed, the front side resist was removed using oxygen plasma etching. Following the dry etching process, the chips were fully released in $\mathrm{HF}: \mathrm{HCl}(1: 1)$ solution with an etch rate of $0.5 \mathrm{micron} / \mathrm{minute}$. After the chips were released, they were dried using a critical point dryer (Leica CPD). The scanning electron microscope (SEM) images of the fabricated device are presented in Figure $3 \mathrm{a}$.

\section{MODEL DERIVATION}

In this study we integrated the levitation based electrode configuration into a MEMS microphone for the first time. Figure 1 shows the electric field distribution of the unit cell of the electrode configuration, which consists of three fixed (red-green-red) and one moving electrodes (gray). The red electrodes are biased while the gray and green electrodes are grounded. This electrode arrangement breaks the symmetry of the electric field of the conventional electrode configuration and creates a net force on each moving electrodes that are attached to the diaphragm. This electrostatic force lifts the diaphragm away from the substrate and prevents the possibility of the pull-in failure. In fact, the green electrode acts as a shield to the bottom surface of the moving (gray) electrode and creates a net force in the upward direction. The thin diaphragm is supported by two short beams such that it can rotate in response to arriving sound waves. The area of the diaphragm is approximately $1 \mathrm{~mm}^{2}$ and the thickness is around $2 \mu \mathrm{m}$. The sensing electrodes are attached to three sides of the diaphragm. Underneath these sensing electrodes there are fixed electrodes having the same voltage as the moving electrodes and the diaphragm. These electrodes are positioned between two bias fixed electrodes, see Figure $3 b$. The dimensions for the microphone design are given in Table I.

The finite element analysis (FEA) simulations show that the first mode of the diaphragm is a rocking mode with a natural frequency of $1510 \mathrm{~Hz}$. However, this model does not include the mass of the electrodes, which shifts the frequency to 1483 $\mathrm{Hz}$. The Figure 5 shows the schematic of the simplified motion of the diaphragm and the air vent. The same figure depicts some of the parameters that are used in the mathematical model. On the bottom of the figure one can see the fabricated chip with and without the thin diaphragm. The mathematical modeling starts with estimating the electrostatic force exerted on the moving electrodes by the applied bias voltage. As mentioned in Section I, the bias voltage is necessary to obtain electronic output from the read-out circuit. Because the electrostatic force acts to levitate, or repel the diaphragm away from the substrate, it also provides a static displacement that increases the travel range of the diaphragm. This property of the levitation based electrode configuration eliminates the possibility of pull-in failure. More description of the working principle of the levitation (repulsive) configuration can be found in reference [10]. To estimate the electrostatic force, we modeled a unit cell of electrodes in the COMSOL finite element analysis software. We can obtain the capacitance as a function of the gap between the moving and fixed (middle) electrode. In Figure 6a, the capacitance of the finger design is presented and the inset of the same figure has the dimensions for the electrode design. After the capacitance is estimated and plotted we fit a seventh order polynomial to obtain an expression for it. One can express the curve fit function as:

$$
C(z)=N_{t} L_{f} \sum_{n=0}^{7} \Gamma_{n} z^{n}
$$

where $\Gamma_{n}$ is the nth term of the coefficient obtained from the polynomial fit, $N_{t}$ is the total number of electrodes, $L_{f}$ is the length of the electrodes. If the function of capacitance is known, electrostatic force can be expressed as:

$$
F_{e s}(z)=\frac{V^{2}}{2} \frac{d C(z)}{d z}
$$

where $V$ is the applied bias voltage and the $\frac{d C(z)}{d z}$ is the change of capacitance. After estimating the force, the next step is to define the motion of the diaphragm. The microphone diaphragm rotates around its axis of support in response to the sound pressure. However, this motion is relatively small and we can simplify the model further and assume a piston-like behavior as depicted in Figure 5. A detailed description of the modeling approach can be found in [4]. In this case, the response of a diaphragm exposed to an incident sound wave can be expressed as

$$
m \ddot{z}+k z+C_{0} \dot{z}=-P A+F_{e s}
$$

where $m, k$ and $C_{0}$ are the effective mass of the diaphragm, mechanical stiffness and the damping coefficient, respectively. 
(a)

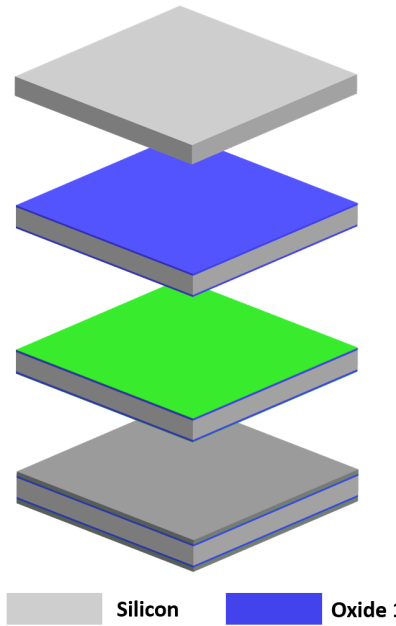

(e)

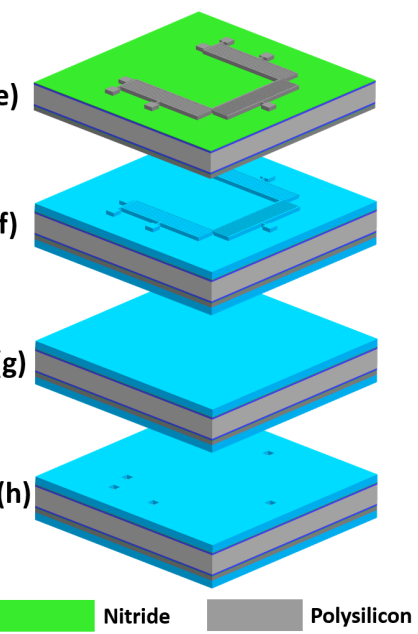

(i)

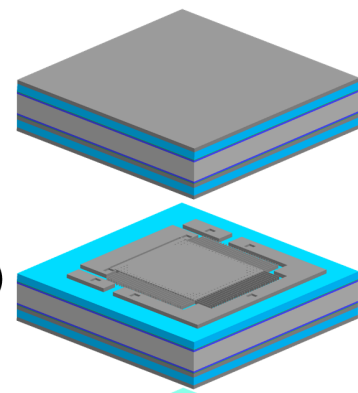

(k)

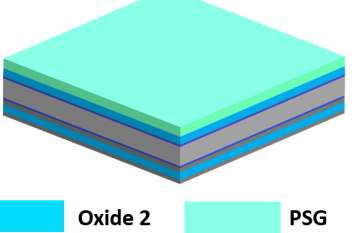

(I)

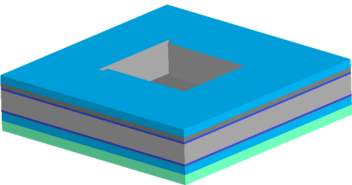

(m1)

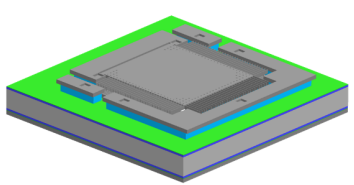

(m2)

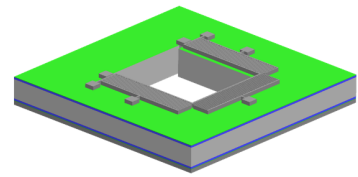

Fig. 2: Fabrication process flow (not to scale): (a) Single side polished 4-inch silicon wafer. (b) LPCVD oxide growth. (c) Low-stress silicon nitride deposition. (d) LPCVD polysilicon deposition. (e) Patterning and dry etching of polysilicon to create fixed electrodes. (f) Sacrificial oxide layer deposition. (g) Planarization of excessive oxide. (h) Patterning anchor openings and dry etching of oxide. (i) Second LPCVD polysilicon deposition. (j) Patterning and dry etching of polysilicon to create diaphragm attached with moving electrodes. (k) Protective PECVD PSG layer deposition. (l) Backside patterning and deep silicon etching to form a back volume. (m1) Diaphragm release and critical-point-drying. (m2) The diaphragm is removed to show the backside opening.
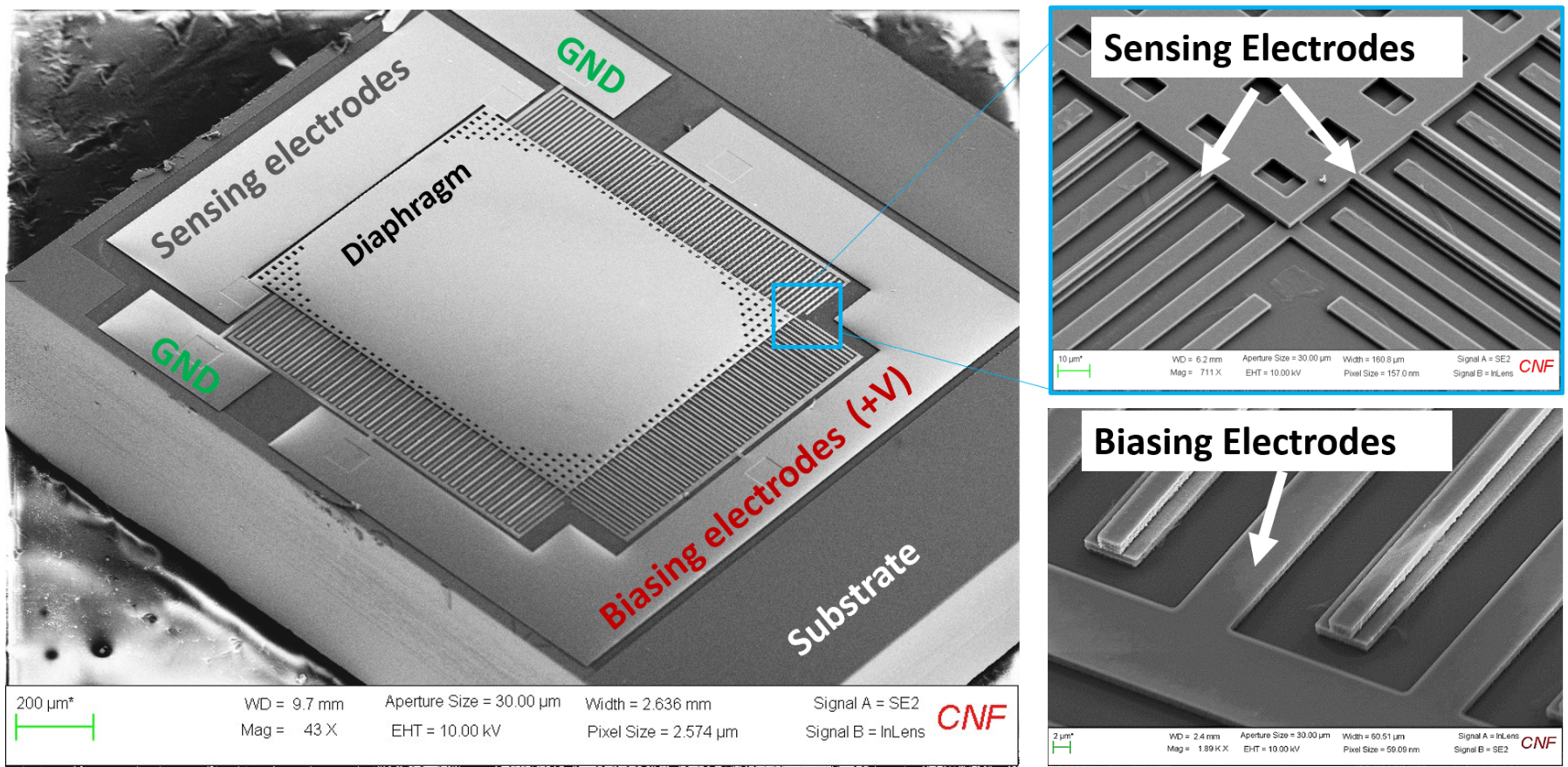

Fig. 3: The figure shows the fabricated chip with biased and sensing electrodes. On the left image a fully released chip is presented. On the right, closer view of the electrodes are depicted.

The mechanical stiffness and damping coefficients may be estimated by $k=4 \pi^{2} f^{2} m$ and $C_{0}=2 \zeta \sqrt{k \cdot m}$, respectively, where $f$ is the natural frequency of the diaphragm which is obtained from the finite element simulations and $\zeta$ is the damping ratio. $P$ is the pressure from the incoming sound wave, $A$ is the effective surface area of the diaphragm and $F_{e s}$ is the electrostatic force. Equation 3 is used to predict the motion of the diaphragm without including any effect from the air vents and back volume. However, the volume under the diaphragm introduces significant stiffness to the system because of its spring-like behavior. The stiffness is inversely proportional to the size of the volume. In addition, the air vent openings around the diaphragm affect the low-frequency cutoff of the microphone response. After including these effects 

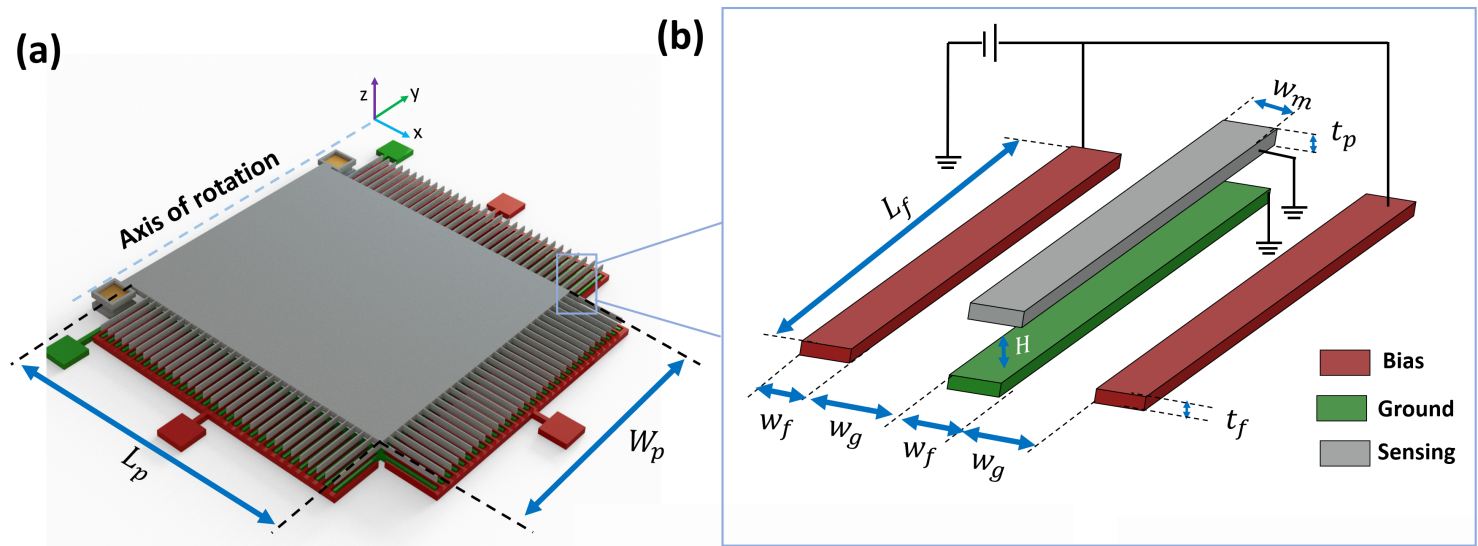

(c)

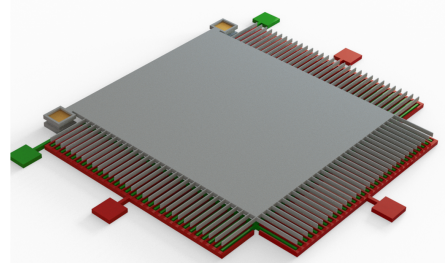

(Rest Position, $V_{\text {bias }}=0$ )

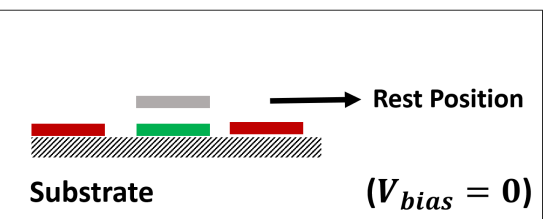

(d)
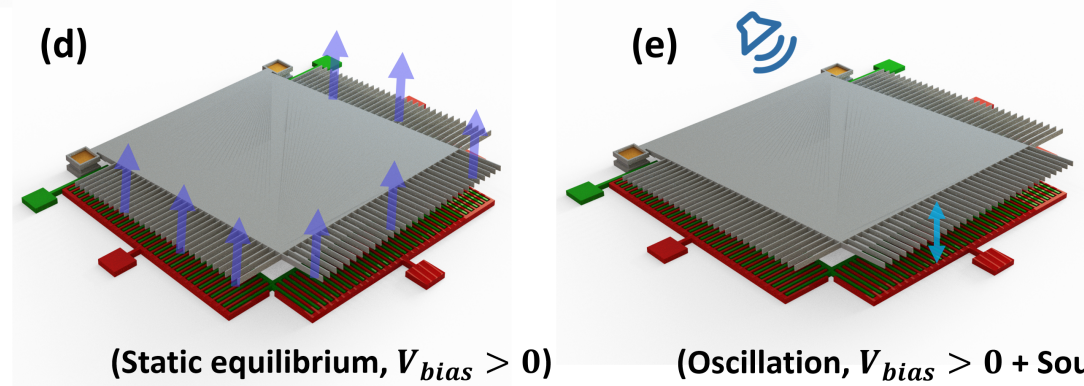

(Oscillation, $V_{\text {bias }}>0+$ Sound )
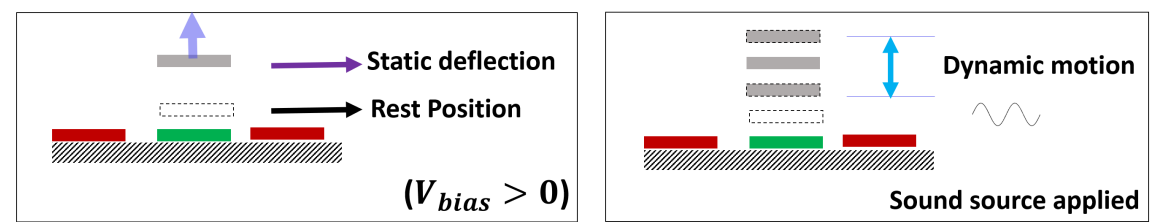

Fig. 4: The figure shows the 3-D model of the proposed microphone design and the operation principle. (a-b) 3-D model of the device with the design parameters. (c) In its rest position the released device is expected to have a $2 \mu \mathrm{m}$ thick initial gap (H). (d) A DC bias is applied to the biasing electrodes, which creates electrostatic force on the fingers and lifts the diaphragm away from the substrate. This is the static equilibrium position of the diaphragm. (e) After the bias is applied the diaphragm is exposed with a sound source, which causes the diaphragm to oscillate around its equilibrium point. This motion can be detected and converted into electrical signals via an electronic circuitry.

the equation becomes coupled as [4]:

$$
\begin{aligned}
& m \ddot{z}+\left(k+K_{d}\right) z+\left(K_{a d}\right) z_{a}+C_{0} \dot{z}=-P A+F_{e s} \\
& m_{a} \ddot{z_{a}}+\left(K_{a a}\right) z_{a}+\left(K_{d a}\right) z+C_{v} \dot{z}_{a}=-P A_{a}
\end{aligned}
$$

where $m_{a}$ is the mass of the air in the air vents and openings. $A_{a}$ is the total area of the air vents. $C_{v}$ represents the squeeze film damping caused by the air slits. $K_{d}, K_{a d}, K_{a a}, K_{d a}$ are stiffness contributions due to back volume and air vents. Table II gives these parameters. One can solve Equation 4 to predict the mechanical response $\left(S_{P Z}\right)$ of the microphone by assuming harmonic behavior such as $z=\bar{Z} e^{i \omega t}$ and $P=$ $\bar{P} e^{i \omega t}$ where $\omega$ is the driving frequency. The transfer functions that predict the mechanical and acoustical sensitivities can also be estimated by

where

$$
S_{P Z}(\omega)=\frac{\bar{Z}}{\bar{P}}=\frac{R_{1}}{R_{2}+R_{3}}
$$

$$
\begin{aligned}
& R_{1}=A_{a} K_{a d}-A \times\left(K_{a a}+i \omega C_{v}-\omega^{2} m_{a}\right) \\
& R_{2}=-K_{a d} K_{d a} \\
& R_{3}=\left(K_{e f f}+i \omega C_{0}-\omega^{2} m\right) \times\left(K_{a a}+i \omega C_{v}-\omega^{2} m_{a}\right)
\end{aligned}
$$

where $K_{e f f}=\left(k+K_{d}-\frac{V^{2}}{2} \frac{d^{2} C}{d z^{2}} \mid\left(z_{0}\right)\right)$. In this equation, $z_{0}$ is the static equilibrium position that can be calculated by dropping the time dependent variables in Equation 3 and solving $k z-\frac{V^{2}}{2} \frac{d C(z)}{d z}=0$. Given the static equilibrium point and the capacitance function one can obtain the electrical response $\left(S_{P z_{0}}\right)$ and acoustic response $\left(S_{z_{0} V}\right)$ :

$$
\begin{aligned}
& S_{z_{0} V}(\omega)=-\left(1 / C_{f}\right) \cdot V \frac{d C}{d z} \mid z_{0} \\
& S_{P z_{0}}(\omega)=S_{P z_{0}} \times S_{z_{0} V}
\end{aligned}
$$



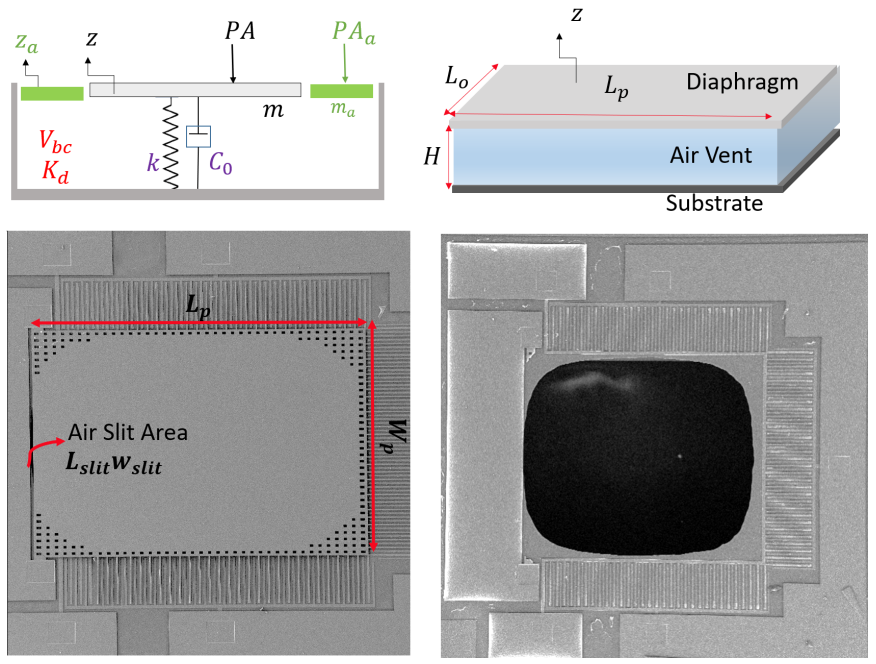

Fig. 5: (Upper left) Schematical representation of the diaphragm motion for an enclosed back chamber [4].(Upper right) Shows the parameters used for air vent under the electrodes. (Bottom) Top view of the microphone with and without the diaphragm.

\begin{tabular}{llll} 
Description & Parameter & Value & Unit \\
\hline Elastic Modulus & $\mathrm{E}$ & 160 & $\mathrm{GPa}$ \\
Density & $\rho$ & 2320 & $\mathrm{~kg} / \mathrm{m}^{3}$ \\
Poisson's Ratio & $\mathrm{v}$ & 0.22 & - \\
Diaphragm width & $\mathrm{W}_{\mathrm{p}}$ & 960 & $\mu \mathrm{m}$ \\
Diaphragm length & $\mathrm{L}_{\mathrm{p}}$ & 1063 & $\mu \mathrm{m}$ \\
Finger length & $\mathrm{L}_{\mathrm{f}}$ & 200 & $\mu \mathrm{m}$ \\
Moving finger width & $\mathrm{w}_{\mathrm{m}}$ & 3.4 & $\mu \mathrm{m}$ \\
Fixed finger width & $\mathrm{w}_{\mathrm{f}}$ & 7.7 & $\mu \mathrm{m}$ \\
Fixed finger separation & $\mathrm{w}_{\mathrm{g}}$ & 8.3 & $\mu \mathrm{m}$ \\
Diaphragm thickness & $\mathrm{t}_{\mathrm{p}}$ & 2 & $\mu \mathrm{m}$ \\
Finger thickness & $\mathrm{t}_{\mathrm{f}}$ & 2 & $\mu \mathrm{m}$ \\
Air gap & $\mathrm{H}_{\mathrm{m}}$ & 2 & $\mu \mathrm{m}$ \\
Spring thickness & $\mathrm{t}_{\mathrm{s}}$ & 2 & $\mu \mathrm{m}$ \\
Spring width & $\mathrm{w}_{\mathrm{s}}$ & 40 & $\mu \mathrm{m}$ \\
Spring length & $\mathrm{L}_{\mathrm{s}}$ & 10 & \\
Total Number of electrodes & $N_{t}$ & 77 & $\mathrm{~N} / \mathrm{m}$ \\
Capacitance Constant & $\Gamma_{0}$ & $2.3233 \times 10^{-11}$ & $\mathrm{~N} / \mathrm{m}^{2}$ \\
Capacitance Constant & $\Gamma_{1}$ & $7.7645 \times 10^{-7}$ & $\mathrm{~N} / \mathrm{m}^{3}$ \\
Capacitance Constant & $\Gamma_{2}$ & $7.47971 \times 10^{-2}$ & $\mathrm{~N} / \mathrm{m}^{4}$ \\
Capacitance Constant & $\Gamma_{3}$ & $3.42989 \times 10^{3}$ & $\mathrm{~N} / \mathrm{m}^{5}$ \\
Capacitance Constant & $\Gamma_{4}$ & $-8.78661 \times 10^{7}$ & $\mathrm{~N} / \mathrm{m}^{6}$ \\
Capacitance Constant & $\Gamma_{5}$ & $1.257413 \times 10^{12}$ & $\mathrm{~N} / \mathrm{m}^{7}$ \\
Capacitance Constant & $\Gamma_{6}$ & $-9.05615 \times 10^{15}$ & $\mathrm{~N} / \mathrm{m}^{8}$ \\
Capacitance Constant & $\Gamma_{7}$ & $2.34822 \times 10^{19}$ & \\
& & & \\
\hline
\end{tabular}

TABLE I: Dimensions for the fabricated device.

\section{EXPERIMENTAL SETUP}

The fabricated chips were attached to a printed-circuit-board using UV curable adhesive (Dymax Ultra Light-Weld). The circuit board had a $1 \mathrm{~mm}$ diameter through hole at the center, which is aligned with the chip backside hole to increase the effective back volume of the microphone. Then, the chips were wire-bonded to the circuit board using an aluminum wedge bonder (Westbond 7400A Ultrasonic Wire Bonder). Also, we
Parameter Variable

\begin{tabular}{ll}
\hline & \\
$m$ & $\rho t_{p} W_{p} L_{p}$ \\
$m_{a}$ & $\rho_{0}\left(d_{\text {slit }} L_{\text {slit }} w_{\text {slit }}+z L_{o}\left(2 L_{p}+W_{p}\right)\right)$ \\
$A_{a}$ & $L_{\text {slit }} w_{\text {slit }}+z\left(2 L_{p}+W_{p}\right)$ \\
$A$ & $L_{p} W_{p}$ \\
$k$ & $4 \pi^{2} f^{2} m$ \\
$K_{d}$ & $\rho_{0} c^{2} A^{2} / V_{b v}$ \\
$K_{a a}$ & $\rho_{0} c^{2} A_{a}^{2} / V_{b v}$ \\
$K_{a d}$ & $\rho_{0} c^{2} A_{a} A / V_{b v}$ \\
$K_{d a}$ & $\rho_{0} c^{2} A_{a} A / V_{b v}$ \\
$C_{0}$ & $2 \zeta \sqrt{k m}$ \\
$C_{v}$ & $6 \mu_{a i r} d_{\text {slit }} L_{\text {slit }} / h_{\text {slit }}$ \\
& \\
\hline
\end{tabular}

TABLE II: Variables in Equation 4 where $d_{\text {slit }}$ : depth of air the slit, $L_{s l i t}$ :length of air in the slit, $w_{s l i t}$ : width of the air in the slit, $h_{\text {slit }}$ : half of the width of the air in the slit, $\mu_{\text {air }}$ is the air viscosity. $L_{o}$ is the length of the diaphragm that overlaps the back volume opening. $V_{b v}$ : back volume size

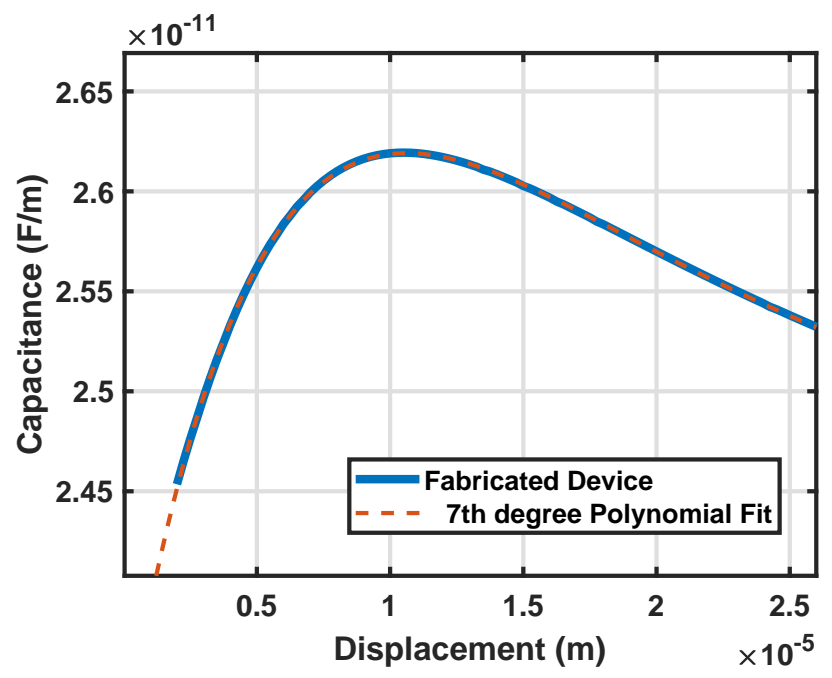

(a) Simulated capacitance function for a unit cell of electrodes. 7th order polynomial is used to curve fit.
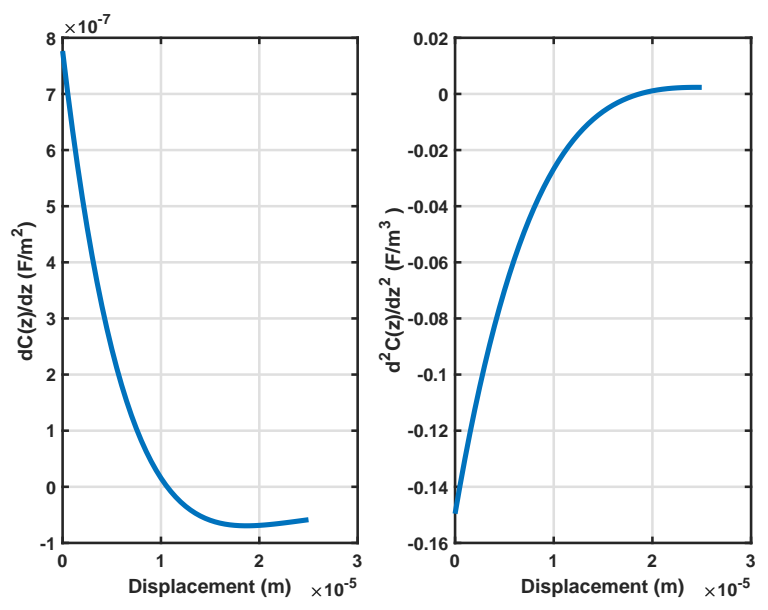

(b) First and second order derivatives of the capacitance.

Fig. 6: COMSOL simulations for the fabricated electrode geometry. 
used a thick aluminum plate to hold the circuit board during the measurements securely. The experiments were performed in the anechoic chamber at Binghamton University. Figure 7 presents the complete setup and the schematic representation for the tests. The experiment started with applying DC voltage to the biasing electrodes, which introduced a static deflection $\left(z_{0}\right)$ to the diaphragm and increased the initial gap between the moving and the fixed electrodes $\left(\mathrm{H}+z_{0}\right)$. After the diaphragm was deflected from its rest position, the sound pressure was created using a loudspeaker. We swept a broad range of pure tone signals $(100 \mathrm{~Hz}-20 \mathrm{kHz})$. The incident pressure was measured close to the MEMS device using a Bruel \& Kjaer 4138 reference microphone. The mechanical response of the diaphragm was measured by a Polytec laser vibrometer consisting of an OFV-534 compact sensor head and an OFV5000 vibrometer controller. The electronic output from the chip was detected via a charge amplifier read-out circuit that consisted of an operational amplifier (OPA 657), a capacitor (1 $p F)$, and feedback-resistors (10 G $\Omega$ ). The output is amplified using a low noise amplifier to increase the quality of the signal. All signals were sent and acquired by National Instruments PXI-1033 Data Acquisition System integrated with the data acquisition toolbox of MATLAB.

\section{RESUlts AND Discussion}

We tested three different chips that are selected from various locations of a wafer. In these experiments, we examined the acoustic performance of the microphones that employ the levitation based electrode design. Performance tests include measuring electrical and acoustic sensitivities. We measured the mechanical response of these microphones for various bias voltages using a laser vibrometer. Figure 8 shows good agreement between experimental and simulation results for the mechanical response to input pressure $\left(S_{P Z}-\mu \mathrm{m} / \mathrm{Pa}\right)$. The results are presented for 40,60, and 80 Volts bias and they show a stiffening effect due to increasing effective stiffness, which is defined by $\left(\left(k+K_{d}-\frac{V^{2}}{2} \frac{d^{2} C}{d z^{2}} \mid\left(z_{0}\right)\right)\right)$. The main parameter that causes this increase is the second derivative of capacitance $\left(\frac{d^{2} C}{d z^{2}} \mid\left(z_{0}\right)\right)$, which is a negative value for the levitation electrode system as seen from Figure 6b. Even though we simplified the dynamic model, it was still complicated to estimate all parameters presented in Table II. The fabricated device had unexpected fabrication related imperfections due to the residual stress on the diaphragm. Some examples to the effect of the stress could be buckled diaphragm and the curled electrodes, which are not trivial to reflect in the mathematical model. For the simulations, to compensate these imperfections we tuned two parameters to predict the results: effective mass $\left(m_{e f f}\right)$ and the stiffness effect of air openings on the diaphragm $\left(K_{a d}\right)$. Also, to estimate the low-frequency cut-off slope correctly, we did trial and error for the stiffness $\left(K_{a d}\right)$. We estimated the effective area to be $\left(A_{a} / 2\right)$ for this parameter. This information is also a valuable reference for future studies such that we can obtain flatter frequency response by adequately controlling the air openings. The natural frequency of the diaphragm $(f)$ is obtained from the FEA analysis, as mentioned in the modeling section. We estimated the damping coefficient $(\zeta)$ from the experiments. Other parameters, such as diaphragm length, finger widths, etc. were obtained from the detailed SEM images of the microphone chips.

In Section III we showed that the acoustic sensitivity of a MEMS microphone is the product of mechanical and electrical outputs $\left(S_{P V}(\omega)=S_{P Z} \times S_{Z V}\right)$. Thus, to evaluate the performance of the devices, we measured mechanical, electrical, and acoustic sensitivities at the same time, which are presented in Figures 9 and 10. Unlike the conventional parallel plate capacitors, the proposed electrode scheme undergoes spring hardening effect. The Figure $9 \mathrm{a}$ shows that as the bias voltage changes from 42 to 102 Volts the mechanical sensitivity decreases due to the increasing stiffness. According to the results the resonance frequency shifted from $2 \mathrm{kHz}$ at 42 Volts to $3.5 \mathrm{kHz}$ at 102 Volts. The Figure 9b presents the electrical response of the microphone. The electrical sensitivity improves with the bias because it is the product of applied bias and the first derivative of capacitance at a static equilibrium point (Eq. 6). Figure 9c shows that the maximum measured sensitivity was around $160 \mathrm{mV} / \mathrm{Pa}$ at $1 \mathrm{kHz}$ and 57 Volts bias.

The experimental results indicate that the microphone worked for a wide range of bias voltages (27-200 Volts) without any pull-in failure. They also reveal that the acoustic sensitivity increased up to a specific bias voltage, which suggests that there is an optimum bias to achieve the highest acoustic sensitivity. However, our earlier studies proposed that the acoustic sensitivity improved as bias voltage changed from 40 to 100 Volts [13], [14]. To explain the difference between the two studies, we need to investigate electrical and mechanical sensitivities individually. In terms of design, the major difference between the presented and earlier microphones was the size of the back volume, which has a significant effect on the effective stiffness of the system. In the earlier studies, the back volume was much smaller compared to the present work. The smaller the back volume is, the larger the stiffness, which reduces the mechanical sensitivity of the microphone drastically. Thus, when the back volume stiffness $\left(K_{d}\right)$ highly dominates the effective stiffness, the contribution of mechanical response to the acoustic sensitivity becomes almost negligible. However, the electrical sensitivity keeps increasing because of the change of the bias voltage, which explains the continuously increasing acoustic sensitivity. It is worth to mention that even though it tends to improve with the bias, the amplitude is much smaller for the smaller back volumes. Thus, we can comment that improving the overall sensitivity with the bias voltage comes at the cost of a smaller sensitivity [14].

The Figure 10 shows the electrical and acoustic sensitivities of three different microphones with the applied bias from 27 to 200 Volts. The results show that the electrical sensitivities tend to increase with the bias while the acoustic sensitivity does not, which can be explained with the above discussed analysis on the sensitivity. For the experiment (Test620), the microphone was applied 200 Volts bias and the sensitivity was measured around $16.1 \mathrm{mV} / \mathrm{Pa}$ at $1 \mathrm{kHz}$. The resonance frequency for this chip was observed around $4922 \mathrm{~Hz}$. For the experimental results presented in Figure 10, even though the trends are very consistent, the amplitude of the sensitivities 

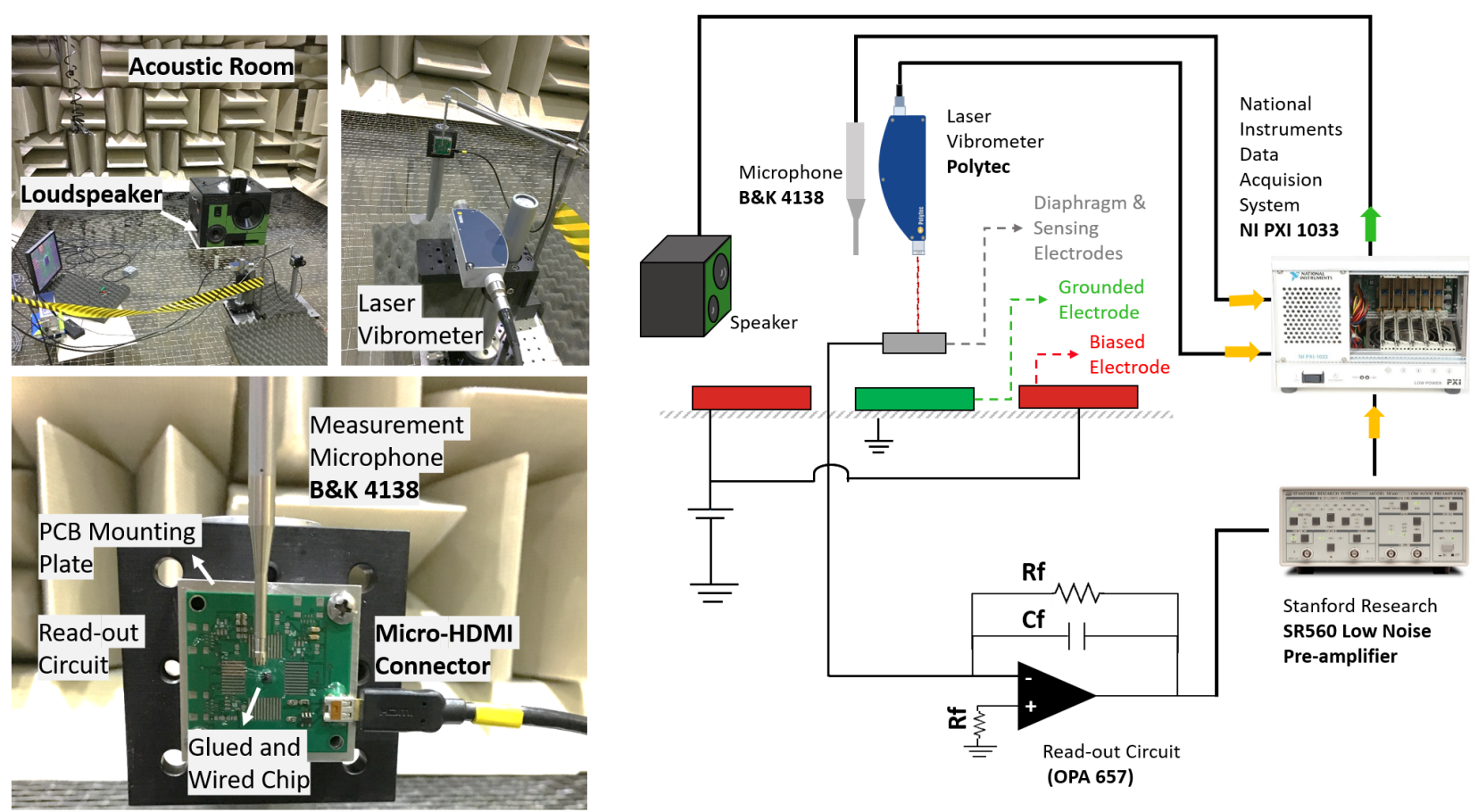

Fig. 7: The chips were tested inside the acoustic core of Binghamton University. The actual setup is shown on the left while a more detailed schematic is drawn on the right.

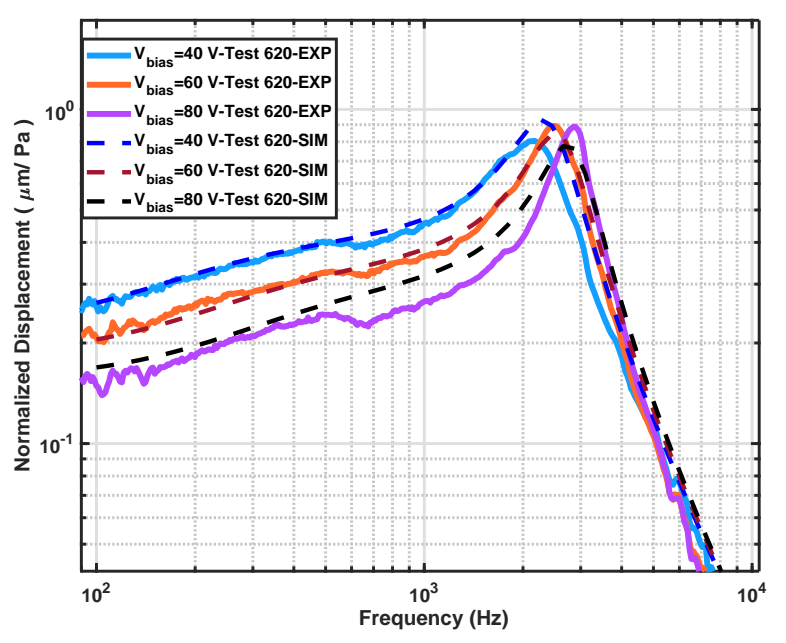

Fig. 8: Simulation results agree well with the experimental results for the mechanical response of the diaphragm. Dashed lines represent the simulation. Solid lines are experimental results. Parameters used for the simulation: $m=1.096 \times 10^{-8} \mathrm{~kg}, f=1483 \mathrm{~Hz}, \rho_{0}=1.206 \mathrm{~kg} / \mathrm{m}^{3}$, $\zeta=0.355, \mu_{\text {air }}=1.846 \times 10^{-5} \mathrm{~kg} /(\mathrm{ms}), L_{o}=100 \mu \mathrm{m}$, $w_{\text {slit }}=5 \mu \mathrm{m}, d_{\text {slit }}=6 \mu \mathrm{m}, L_{\text {slit }}=980 \mu \mathrm{m}$, $V_{b v}=1.75 \times 10^{-7} \mathrm{~m}^{3}$.

are different for each chip. According to our observations, one of the drawbacks of this preliminary microphone prototype is that the response is dependent on the fabrication parameters. For example, some residual stress on the polysilicon film may curl the moving electrodes up or down, which totally changes the electric field and the resultant electrostatic force on the electrodes. This change will have a direct impact on the microphone response. Another example is the back volume opening. As seen at the bottom right of Figure 5, the back volume has a slightly oval shape. However, the actual mask design for the backside through etching was a rectangular shape with some critical boundaries. Due to high aspect ratio DRIE etching of silicon, we were unable to obtain consistent back volume openings at the back of the chips, which changes the effective area. To overcome this issue, we believe that having a circular shape diaphragm and back volume will provide better performance.

Having demonstrated that the levitation electrode configuration can be utilized in a microphone design, we have also explored in what ways this alternate configuration can facilitate the creation of improved designs. It is well-known that the use of a diaphragm having minimal mass and stiffness will lead to improved frequency response and bandwidth [15]. Unfortunately, a diaphragm having too much compliance cannot be used in conventional parallel-plate configurations because the stiffness of the diaphragm must be high enough to avoid it being pulled in to the backplate electrode by the attractive electrostatic force. In the present study, the use of electrodes that levitate, or push on the diaphragm rather than pull it toward the biasing electrode opens up the possibility of using moving electrodes having nearly arbitrary mechanical compliance because the electrostatic force will never cause it to collapse against the biasing electrode. This will allow greatly improved bandwidth and sensitivity. To explore the possibility of employing a highly compliant microphone diaphragm we implemented the same design procedure to a thin- 


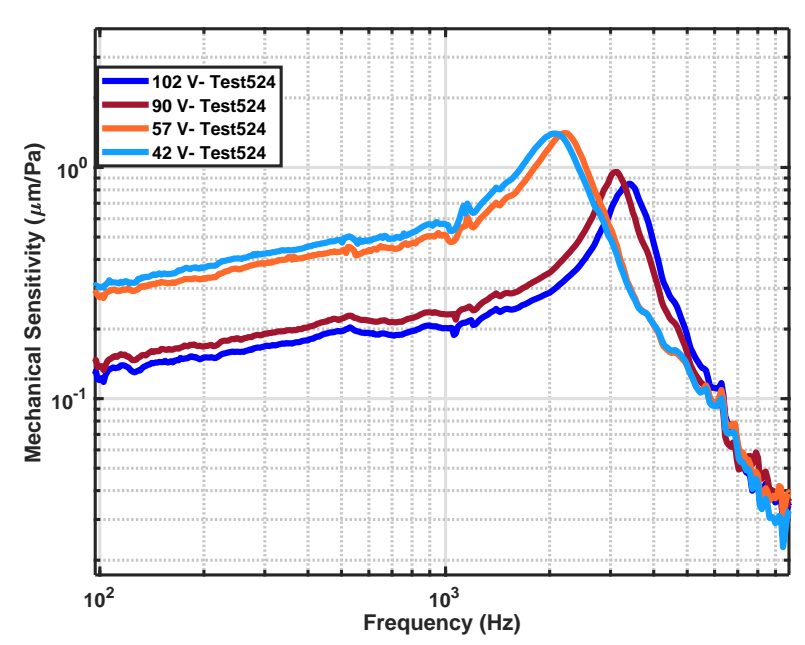

(a) Measured mechanical sensitivity of the microphone for various DC voltages.

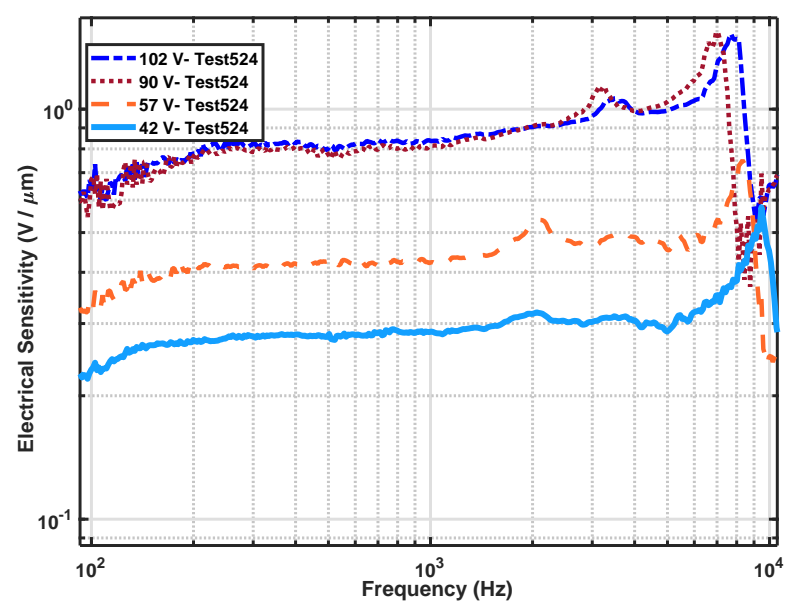

(b) Measured electrical sensitivity of the microphone for various DC voltages.

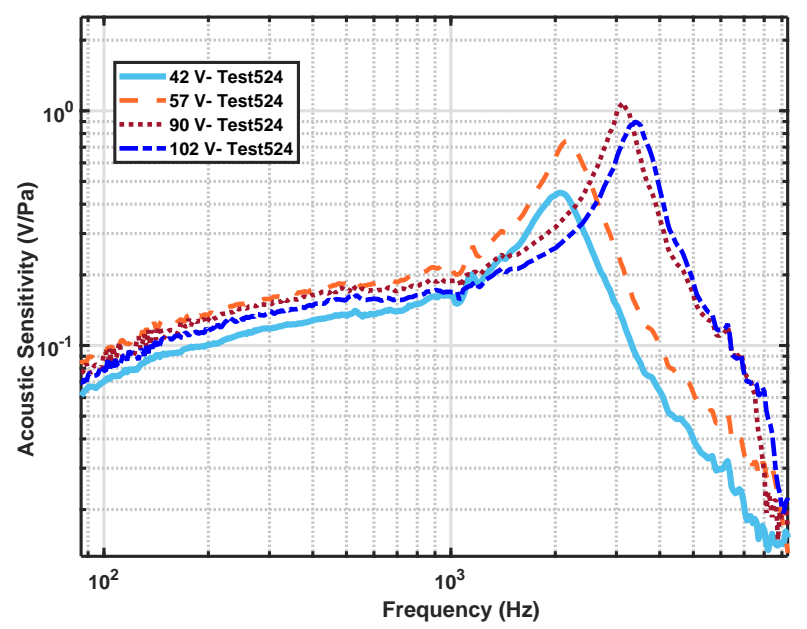

(c) Measured acoustic sensitivity of the microphone for various DC voltages.

Fig. 9: The Mechanical, acoustic and electrical sensitivities of a MEMS microphone with radical change on the electrode design.
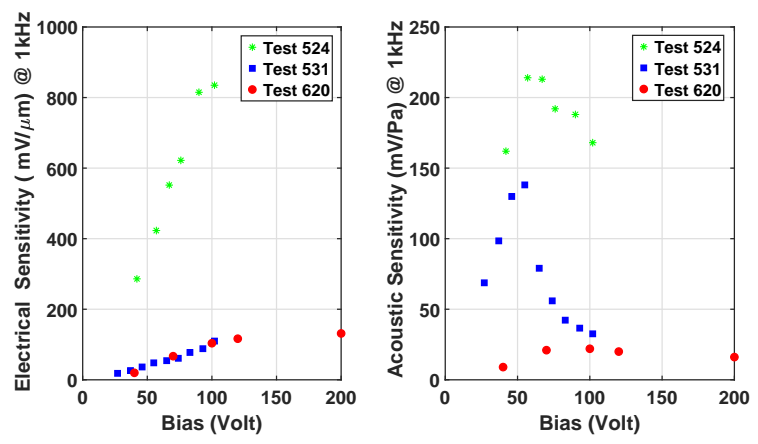

Fig. 10: Electrical and acoustic sensitivities at $1 \mathrm{kHz}$ for three different chips are presented. *: Chip 1, $\mathbf{\square}$ : Chip 2, • : Chip 3.

ner polysilicon diaphragm with attached moving electrodes. Our goal was to make the diaphragm thin enough that it will tend to move with the air as much as possible due to its high compliance. In general, this is the ultimate goal for building high sensitivity acoustic sensors [4], [15]. The size of the diaphragm was assumed to be $1 \mathrm{~mm} \times 1 \mathrm{~mm} \times 0.4 \mu \mathrm{m}$ with a $525 \mu \mathrm{m}$ deep back volume. The supporting beams were a little longer and wider than the one we had in the present study (30 $\mu \mathrm{m} \times 30 \mu \mathrm{m} \times 0.4 \mu \mathrm{m})$. Based on the changes on the dimensions we simulated a new scenario for the electrostatic force. We kept the rest of the design parameters the same as the present study. The expected natural frequency was found to be around $250 \mathrm{~Hz}$ according to our FEA simulations. However, because of the low compliant back volume this frequency was pushed to around $70 \mathrm{kHz}$. Using the same mathematical model in Section III, the acoustic sensitivity for various DC values $(20-300 \mathrm{~V})$ were predicted and presented in Figure 11. These promising results indicate that one can implement the levitation based electrode design to a much thinner plate design and increase the sensitivity without any pull-in concern by simply increasing the DC bias. This also agrees well with the analysis that was discussed above, which mentioned that dominant back volume stiffness enables us to increase the acoustic sensitivity with bias voltage even though the amplitude is smaller compared to less stiff cases. Being able to avoid pull-in failure enables the design of much more compliant and acoustically sensitive diaphragms which will lead to improved performance.

\section{CONCLUSION}

In this study, we presented a MEMS capacitive microphone that can utilize high bias voltages (27 - 200 Volts) and keep functioning without any failure. We tested the mechanical and electrical performance of these microphones in an acoustic chamber using a laser vibrometer and a read-out circuit. The experiments and the analytical model showed that one could design an acoustic sensor with a radical change of the electrode design and improve the sensitivity simply by increasing the bias voltage without any concern of pull-in failure. The use of higher bias voltages helps to lower the noise and increase the signal to noise ratio. However, for capacitive 


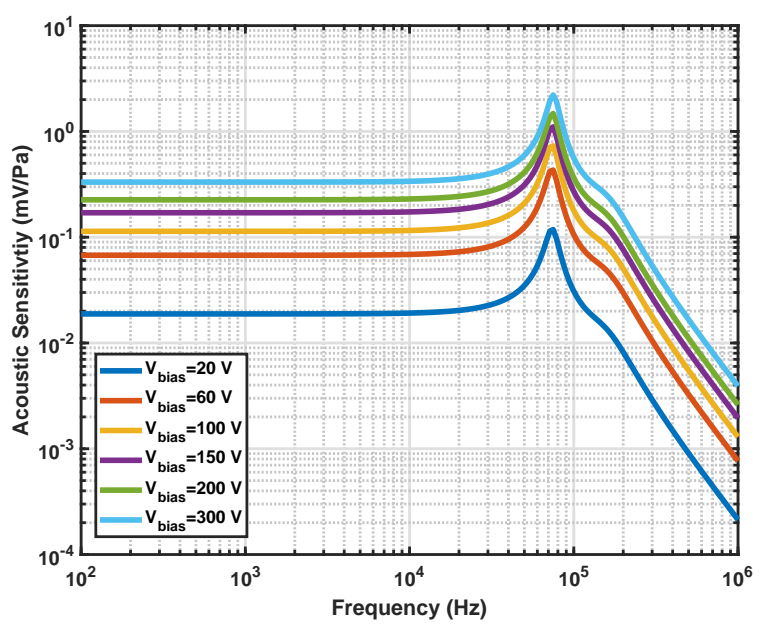

Fig. 11: Acoustic sensitivity for the proposed future design. Diaphragm is designed to be made of $0.4 \mu \mathrm{m}$ thick polysilicon.

sensors, this has been a major concern due to the pull-in limitation of conventional parallel-plate designs. The levitation based design used here is simple enough to be applied to a wide range of capacitive sensor applications in accelerometers, gyroscopes, filters, and logic gate devices.

\section{ACKNOWLEDGMENT}

The authors would like to acknowledge the financial support of this study by the National Science Foundation (NSF) through grant ECCS1608692. Fabrication of the microphone was performed at Cornell NanoScale Facility, an NNCI member supported by NSF Grant NNCI-1542081.

\section{REFERENCES}

[1] J. Czarny, Thesis: Conception, fabrication and characterization of a MEMS microphone. The University of Lyon, 2015.

[2] M. Royer, J. Holmen, M. Wurm, O. Aadland, and M. Glenn, "Zno on si integrated acoustic sensor," Sensors and Actuators, vol. 4, pp. 357 $362,1983$.

[3] M. A. Shah, I. A. Shah, D.-G. Lee, and S. Hur, "Design approaches of mems microphones for enhanced performance," Journal of Sensors, vol. 2019 , p. 26, 2019

[4] R. Miles, A Physical Approach to Engineering Acoustics. Springer- in press, 2019.

[5] A. Dehé, "Silicon microphone development and application," Sensors and Actuators A: Physical, vol. 133, no. 2, pp. 283 - 287, 2007.

[6] C. Chan, W. Lai, M. Wu, M. Wang, and W. Fang, "Design and implementation of a capacitive-type microphone with rigid diaphragm and flexible spring using the two poly silicon micromachining processes," IEEE Sensors Journal, vol. 11, no. 10, pp. 2365-2371, Oct 2011.

[7] B. A. Ganji, S. B. Sedaghat, A. Roncaglia, and L. Belsito, "Design and fabrication of very small mems microphone with silicon diaphragm supported by z-shape arms using soi wafer," Solid-State Electronics, vol. 148, pp. $27-34,2018$.

[8] R. Miles and R. Hoy, "The development of a biologically-inspired directional microphone for hearing aids," Audiology and Neurotology, vol. 11, no. 2, pp. 86-94, 2006.

[9] R. Miles, W. Cui, Q. Su, and D. Homentcovschi, "A mems lownoise sound pressure gradient microphone with capacitive sensing," Microelectromechanical Systems, Journal of, vol. 24, no. 1, pp. 241248, Feb 2015.
[10] S. He and R. BenMrad, "Large-stroke microelectrostatic actuators for vertical translation of micromirrors used in adaptive optics," Industrial Electronics, IEEE Transactions on, vol. 52, no. 4, pp. 974-983, 2005.

[11] M. Pallay, R. N. Miles, and S. Towfighian, "A tunable electrostatic mems pressure switch," IEEE Transactions on Industrial Electronics, pp. 1-1, 2019.

[12] M. Daeichin, M. Ozdogan, S. Towfighian, and R. Miles, "Dynamic response of a tunable mems accelerometer based on repulsive force," Sensors and Actuators A: Physical, vol. 289, pp. 34 - 43, 2019.

[13] M. Ozdogan and S. Towfighian, "A mems microphone using repulsive force sensors," in ASME. IDETC Conference, Volume 4: 21st Design for Manufacturing and the Life Cycle Conference; 10th International Conference on Micro- and Nanosystems:V004T08A017,August, Aug. 2016.

[14] M. Ozdogan, S. Towfighian, and R. N. Miles, "Fabrication and experimental characterization of a mems microphone using electrostatic levitation," in SENSORS, 2019 IEEE, Oct. 2019.

[15] J. Zhou and R. N. Miles, "Directional sound detection by sensing acoustic flow," IEEE Sensors Letters, vol. 2, no. 2, pp. 1-4, June 2018.

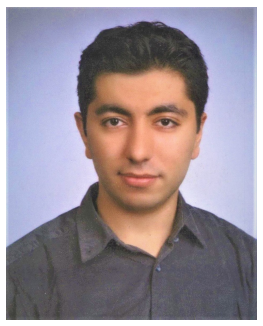

Mehmet Ozdogan received the B.S. degree in mechanical engineering from the Yildiz Technical University, Istanbul, Turkey, in 2012. He received his M.S. degree in mechanical engineering from, SUNY Binghamton, Binghamton, NY, USA, in 2015, where he is currently working toward his Ph.D. degree. His current research interests include fabrication and characterization of MEMS/NEMS devices.

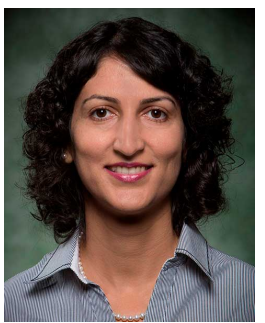

Dr. Shahrzad Towfighian received her B.S. degree from Amirkabir University of Technology, Iran and her M.S. degree from Ryerson University, Canada. She then obtained her Ph.D. degree from the University of Waterloo, Canada. She joined the Mechanical Engineering department at Binghamton University in Fall 2013. She has been the recipient of several grants from the National Science Foundation and the National Health Institute. Her research interests are micro-electro-mechanical systems and energy harvesting for bio-medical devices. She focuses on creating theoretical and experimental frameworks to explain the underlying mechanism of electro-mechanical systems. Using these frameworks, she seeks innovative methods to improve functionality of devices for various applications.

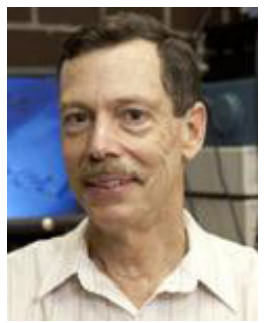

Dr. Ronald Miles received the B.S. degree in electrical engineering from the University of California at Berkeley, Berkeley, CA, USA, and the M.S. and Ph.D. degrees in mechanical engineering from the University of Washington. He has been with the Department of Mechanical Engineering, SUNY Binghamton, Binghamton, NY, USA, since 1989. He has served as the Director of Graduate Studies, the Director of Undergraduate Studies, Associate Chair, Professor, Distinguished Professor, Associate Dean for Research, and currently serves as the Chair of the Department of Mechanical Engineering. 